\title{
Cholinergic deficit in olfactory bulbectomized animals as a model of neurodegenerative diseases
}

\author{
Mikhail Stepanichev \\ IHNA\&NPh RAS, Moscow, Russia \\ mikhail_stepanichev@yahoo.com \\ Anna Manolova \\ IHNA\&NPh RAS, Moscow, Russia \\ ashit@mail.ru
}

\author{
Olga Nedogreeva \\ IHNA\&NPh RAS, Moscow, Russia \\ nedogreewaolga@gmail.com \\ Natalia Gulyaeva \\ IHNA\&NPh RAS, Moscow, Russia \\ nata_gul@mail.ru
}

\author{
Natalia Lazareva \\ IHNA\&NPh RAS, Moscow, Russia \\ nalaza@rambler.ru
}

\begin{abstract}
Cholinergic deficit is a feature of age-related memory decline and age-associated dementia. In the present study we show that olfactory bulbectomy in mice resulted in the development of cognitive impairments and a decrease in the number of cholinergic markers in the medial septal area while the number of neurons remained being stable. The involvement of oxidative stress in the development of cholinergic abnormalities was evident. Thus, olfactory bulbectomy may be used as a model of early stages of neurodegenerative diseases.
\end{abstract}

Keywords - acetylcholine, olfactory bulbectomy, Alzheimer's disease, oxidative stress

\section{Motivation and aim}

\section{Motivation}

Acetylcholine (ACh) is a neurotransmitter playing an important role in higher brain functions, including attention, learning and memory. Cognitive decline found in aging is associated with the development of cholinergic deficit probably because of degeneration of cholinergic neurons. The impairments of the cholinergic system often manifests in patients with dementia, including Alzheimer's disease. However, the number of cholinergic neurons in the basal nucleus of Meynert in aged individuals without cognitive impairments or in patients with mild cognitive impairments was similar to that observed in age-matched controls. Similarly, the development of cholinergic deficit in the absence of neuronal loss could be observed in some animal models of neurodegenerative diseases.

Aim

Here, we studied the time course of the development of behavioral impairments and features of cholinergic deficit in olfactory bulbectomized animals.

\section{Methods}

The experiments were performed in adult Wistar female rats, CD1 female mice and C57B1/6 male mice. The animals were subjected to olfactory bulbectomy (OBX) by aspiration under chloral hydrate anesthesia as described previously.

OBX animals were subjected to series of behavioral tests, including tests for estimation of emotionality and cognitive functions. Immunohistochemical and biochemical methods were used to study the indices of cholinergic system and oxidative stress.

\section{Results}

OBX in female Wistar rats resulted in the appearance of some depressive-like behavioral features, such as the decreased sucrose consumption, hyperactivity, anxiety-like behavioral features impaired short-term memory and an enhancement of avoidance behavior one month after surgery. However, these behavioral abnormalities could be also associated with some disturbances in hippocampal function, which is supported by the presence of cellular changes in this brain structure. We could not find any effects of OBX on the number of cholinergic neurons in the medial septum-diagonal band of Broca as well as the ACh content and acetylcholinesterase activity in the septum, hippocampus, and neocortex. On the contrary, in female CD1 mice OBX impaired spontaneous alternation behavior and decreased the number of cholinergic neurons in the medial septum-diagonal band of Broca. These data demonstrate that OBX does not significantly affect the cholinergic system in rats. We also conclude that rats and mice differentially respond to OBX.

To futher examine the development of cholinergic deficit we studied the effects of OBX in male $\mathrm{C} 57 \mathrm{Bl} / 6$ mice. We revealed that behavioral abnormalities developed in OBX mice starting at lesat two weeks after surgey and remained to be detected until 50 days later on. We revealed a decrease in the number of cholinergic cells in the medial septum-diagonal band of Broca. However, the number of neurons did not change in the OBX mice compared to the control group.

We studied the level of oxidative modifications in nucleic acids using anti-8OHG DNA/RNA antibodies. We did not find any staining in the neuronal nuclei whereas cytoplasm stained intensely. We found that the levels of damaged DNA/RNA staining in the cytoplasm of choline acetyltransferase (ChAT) positive or ChAT negative cells in the medial septum were higher compared to sham-operated mice. Furthermore, the content of oxidatively modified proteins was higher in the hippocampus and medial septum of OBX mice. These data show that oxidative stress may be involved in the development of cholinergic deficit found in OBX animals.

\section{ACKNOWLEDGMENT}

Supported by the RFBR (20-015-00226). 